\title{
Quantum Fisher Information of Bipartitions of $W$ States
}

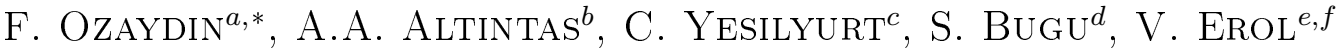 \\ ${ }^{a}$ Department of Information Technologies, Faculty of Science and Letters, Isik University, Istanbul, Turkey \\ ${ }^{b}$ Department of Electrical Engineering, Faculty of Engineering and Architecture, Okan University, Istanbul, Turkey \\ ${ }^{c}$ Department of Computer Engineering, Faculty of Engineering and Architecture, Okan University, Istanbul, Turkey \\ ${ }^{d}$ Department of Computer Engineering, Institute of Science, Istanbul, Turkey \\ ${ }^{e}$ Department of Computer Engineering, Institute of Science, Okan University, Istanbul, Turkey \\ ${ }^{f}$ Provus A MasterCard Company R\&D Center, Istanbul, Turkey
}

\begin{abstract}
We study the quantum Fisher information (QFI) of $W$ states and $W$-like states under decoherence. In particular, we find that on the contrary to amplitude damping and depolarizing decoherence, a $W$-like state of 3 qubits obtained by discarding 1 qubit of a genuine $W$ state of 4 qubits is more robust than a genuine $W$ state of 3 qubits under amplitude amplifying and phase damping decoherence.
\end{abstract}

DOI: 10.12693/APhysPolA.127.1233

PACS: 03.67.-a, 03.65.Ud, 03.65.Yz

\section{Introduction}

Quantum Fisher information (QFI) characterizes the phase sensitivity of a parameter of a system with respect to $\mathrm{SU}(2)$ rotations of the system $[1,2]$. It is a practical tool for detecting multipartite entanglement: If the maximum QFI among the directions averaged over the number of particles, i.e. maximal mean QFI of a state, exceeds 1 (the so-called shot-noise limit), then it is multipartite entangled [3]. (We will use "QFI" for "maximal mean QFI" throughout this paper.) Since the best separable states can achieve the shot-noise limit, such multipartite entangled states can outperform them and are called "useful" states. On the other hand, multipartite entanglement does not imply a QFI larger than 1, in general: There are multipartite entangled states that are not useful even when they are pure [4]. Also the useful states may become un-useful when they are subjected to decoherence due to unavoidable interactions with the environment. For example although an $N$ particle pure Greenberger-Horne-Zeilinger (GHZ) state achieves the fundamental limit on the QFI, i.e. $N$; it cannot achieve even the shot-noise limit when the (amplitude damping/amplifying, depolarizing or phase damping) decoherence is strong enough [5]. Studying the QFI of states in the context of multipartite entanglement and the sensitivity, they can provide under decoherence, is recently attracting more attention in quantum metrology and in quantum information [6-17].

On the other hand, currently there is an intense effort on efficient generation of large-scale photonic $W$ states, yet usually not taking into account the experimental imperfections and photon losses [18-25], where QFI may be extremely helpful. QFI of genuine $W$ states under

* corresponding author; e-mail: mansursah@gmail.com decoherence has been studied in [26].

In this work we study QFI of $W$ states under decoherence as they lose one particle. In particular, we consider a genuine $W$ state of 3 particles and another state (we call " $W$-like state") of 3 particles, which is obtained by discarding 1 particle of a genuine $W$ state of 4 particles. Considering each particle of these two states in the same decoherence channel with the equal decoherence strength, we show that, although genuine $W$ state performs better than the $W$-like state in amplitude damping and depolarizing channels, it is interestingly the opposite when the states are in amplitude amplifying or in phase damping channels: $W$-like state performs better than the genuine $W$ state.

\section{Quantum fisher information}

Maximum mean quantum Fisher information per particle of a mixed quantum state $\rho$ of $N$ particles with the eigenvalues $p_{i}$ and the associated eigenvectors $|i\rangle$, can be obtained by $\lambda_{\max } / N$ [5], where $\lambda_{\max }$ is the largest eigenvalue of the symmetric $C$ matrix with the elements

$$
\begin{aligned}
C_{k l} & =\sum_{i \neq j} \frac{\left(p_{i}-p_{j}\right)^{2}}{p_{i}+p_{j}}\left[\left\langle i\left|J_{k}\right| j\right\rangle\left\langle j\left|J_{l}\right| i\right\rangle\right. \\
& \left.+\left\langle i\left|J_{l}\right| j\right\rangle\left\langle j\left|J_{k}\right| i\right\rangle\right],
\end{aligned}
$$

with the angular momentum operators $J_{n}$ in the $n$th direction defined in terms of the Pauli operators $\sigma_{x, y, z}$

$$
J_{n}=\sum_{\alpha=x, y, z} \frac{1}{2} n_{\alpha} \sigma_{\alpha}
$$

A genuine $W$ state of 3 particles, $\rho^{G}=\left|W_{3}\right\rangle\left\langle W_{3}\right|$ is given as

$$
\left|W_{3}\right\rangle=\frac{|001\rangle+|010\rangle+|100\rangle}{\sqrt{3}},
$$

and the mixed $W$-type state of 3 particles, $\rho^{L}$ obtained by tracing out 1 particle from a genuine $W$ state of 4 particles is given as 


$$
\rho^{L}=\frac{3}{4}\left|W_{3}\right\rangle\left\langle W_{3}\left|+\frac{1}{4}\right| 000\right\rangle\langle 000| .
$$

\section{Decoherence channels}

The basic decoherence channels we consider in this work can be represented by the Kraus operators, i.e.

Amplitude damping,

$$
\left(\begin{array}{cc}
1 & 0 \\
0 & \sqrt{1-p}
\end{array}\right),\left(\begin{array}{cc}
0 & \sqrt{p} \\
0 & 0
\end{array}\right) \text {. }
$$

Amplitude amplifying

$$
\left(\begin{array}{cc}
\sqrt{1-p} & 0 \\
0 & 1
\end{array}\right),\left(\begin{array}{cc}
0 & 0 \\
\sqrt{p} & 0
\end{array}\right) \text {. }
$$

Depolarizing

$$
\begin{aligned}
& \left(\begin{array}{cc}
\sqrt{1-\frac{3 p}{4}} & 0 \\
0 & \sqrt{1-\frac{3 p}{4}}
\end{array}\right),\left(\begin{array}{cc}
0 & \frac{\sqrt{p}}{2} \\
\frac{\sqrt{p}}{2} & 0
\end{array}\right), \\
& \left(\begin{array}{cc}
0 & -\frac{i \sqrt{p}}{2} \\
\frac{i \sqrt{p}}{2} & 0
\end{array}\right),\left(\begin{array}{cc}
\frac{\sqrt{p}}{2} & 0 \\
0 & -\frac{\sqrt{p}}{2}
\end{array}\right) .
\end{aligned}
$$

Phase Damping

$$
\left(\begin{array}{cc}
\sqrt{p} & 0 \\
0 & 0
\end{array}\right),\left(\begin{array}{cc}
0 & 0 \\
0 & \sqrt{p}
\end{array}\right),\left(\begin{array}{cc}
\sqrt{1-p} & 0 \\
0 & \sqrt{1-p}
\end{array}\right) .
$$

Applying the Kraus operators of the decoherence channels to each qubit of $\rho^{G}$ and $\rho^{L}$, we obtain the density matrices of the decohered states. Substituting the eigenvalues and the associated eigenvectors of the states and the angular momentum operators to Eq. 1, it is straightforward to calculate the QFI of the states under each decoherence channels. We find that, as one would intuitively expect, in amplitude damping channel (Fig. 1a) and depolarizing channel (Fig. 1b), $\rho^{G}$ is more robust than $\rho^{L}$ but surprisingly, in amplitude amplifying channel (Fig. 1c) and phase damping channel (Fig. 1d), $\rho^{L}$ turns to be more robust than $\rho^{G}$.

\section{Conclusions}

In conclusion we found that in amplitude amplifying and phase damping channels, a $W$-like state of 3 particles, which is obtained by discarding 1 particle of a genuine $W$ state of 4 particles, is more robust than a genuine $W$ state of 3 particles, in a sense that it provides higher quantum Fisher information (QFI). Since QFI characterizes the phase sensitivity of a state with respect to $\mathrm{SU}(2)$ rotations, our results suggest that in phase sensitive quantum metrology and quantum information processing tasks that require an $\mathrm{N}$ particle state in the $W$ form as resource, if the system is subject to an inevitable amplitude amplifying or phase damping decoherence, rather than a genuine $W$ state of $\mathrm{N}$ particles, it is preferable to use the state obtained by discarding one particle of a genuine $W$ state of $N+1$ particles. Our work may also shed light to the efforts on preparing large-scale $W$ states when particle loss is taken into account.

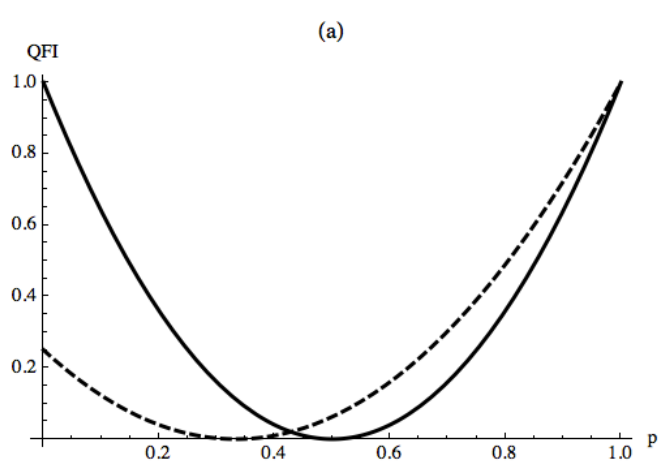

(b)

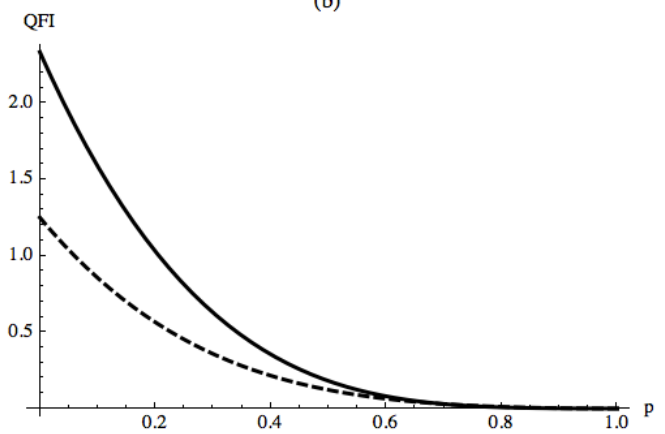

(c)

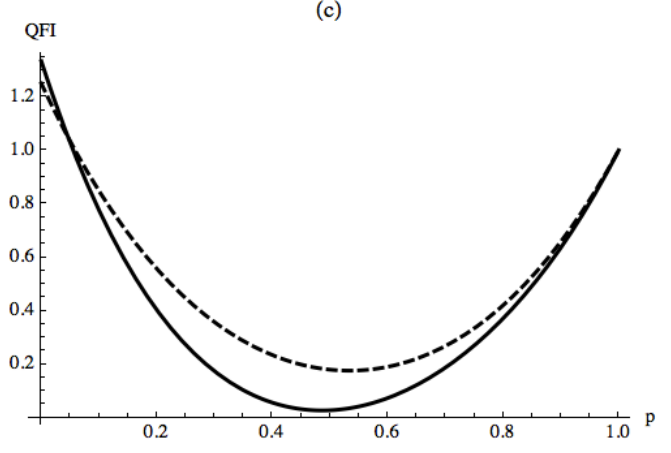

(d)

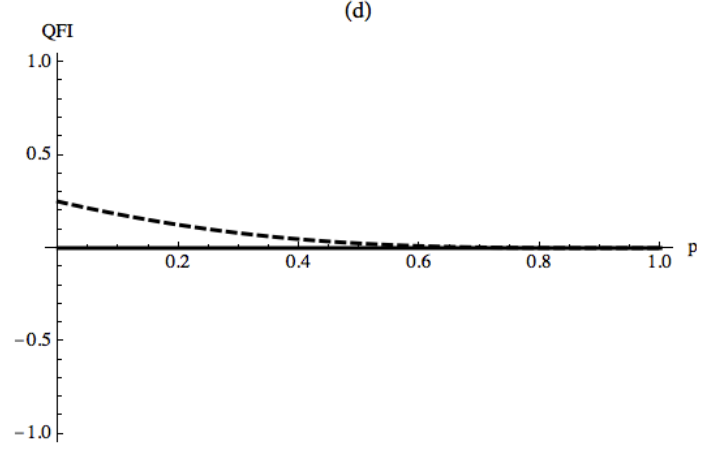

Fig. 1. Quantum Fisher information of genuine $W$ state of 3 particles (solid lines) and $W$-like state of 3 particles, obtained by discarding 1 particle of a genuine $W$ state of 4 particles (dashed lines) under the decoherence channels: (a) amplitude damping, (b) depolarizing, (c) amplitude amplifying, (d) phase damping, with respect to decoherence strength $p$. $W$-like state is more robust than genuine $W$ state in amplitude damping and in phase damping channels. 


\section{Acknowledgments}

This work was funded by Isik University Scientific Research Fund, Grant No. BAP-14A101.

\section{References}

[1] C.W. Helstrom, Quantum Detection and Estimation Theory, Academic Press, New York 1976.

[2] A.S. Holevo, Probabilistic and Statistical Aspects of Quantum Theory, North-Holland, Amsterdam 1982.

[3] L. Pezze, A. Smerzi, Phys. Rev. Lett. 102, 100401 (2009).

[4] P. Hyllus, O. Gühne, A. Smerzi, Phys. Rev. A 82, 012337 (2010).

[5] J. Ma, Y-X. Huang, X. Wang, C.P. Sun, Phys. Rev. A 84, 022302 (2011).

[6] Y. Matsuzaki, S.C. Benjamin, J. Fitzsimons, Phys. Rev. A 84, 012103 (2011).

[7] P. Hyllus, W. Laskowski, R. Krischek, C. Schwemmer, W. Wieczorek, H. Weinfurter, L. Pezze, A. Smerzi Phys. Rev. A 85, 022321 (2012).

[8] G. Toth, Phys. Rev. A 85, 022322 (2012).

[9] F. Ozaydin, A.A. Altintas, S. Bugu, C. Yesilyurt, Int. J. Theor. Phys. 52, 2977 (2013).

[10] A.W. Chin, S.F. Huelga, M.B. Plenio, Phys. Rev. Lett. 109, 233601 (2012).

[11] K. Berrada, S. Abdel-Khalek, A.-S.F. Obada, Phys. Lett. A 376, 1412 (2012).

[12] F. Benatti, S. Alipour, A.T. Rezakhani, New J. Phys. 16, 015023 (2014).
[13] D. Hosler, P. Kok, Phys. Rev. A 88, 052112 (2013).

[14] F. Ozaydin, A.A. Altintas, S. Bugu, C. Yesilyurt, Acta Phys. Pol. A 125, 606 (2014).

[15] F. Ozaydin, A.A. Altintas, S. Bugu, C. Yesilyurt, M. Arik, Int. J. Theor. Phys. 53, 3219 (2014).

[16] X. Jing, J. Liu Jing, W. Zhong, X. Wang, Commun. Theor. Phys. 61, 115 (2014).

[17] V. Erol, F. Ozaydin, A.A. Altintas, Sci. Rep. 4 $5422(2014)$

[18] T. Tashima, S.K. Ozdemir, T. Yamamoto, M. Koashi, N. Imoto, Phys. Rev. A 77, 030302 (2008).

[19] T. Tashima, S.K. Ozdemir, T. Yamamoto, M. Koashi, N. Imoto, New J. Phys. 11, 023024 (2009).

[20] T. Tashima, T. Wakatsuki, S.K. Ozdemir, T. Yamamoto, M. Koashi, N. Imoto, Phys. Rev. Lett. 102, 130502 (2009).

[21] T. Tashima, T. Kitano, S.K. Ozdemir, T. Yamamoto, M. Koashi, N. Imoto, Phys. Rev. Lett. 105, 210503 (2010).

[22] S.K. Ozdemir, E. Matsunaga, T. Tashima, T. Yamamoto, M. Koashi, N. Imoto, New J. Phys. 13, 103003 (2011).

[23] S. Bugu, C. Yesilyurt, F. Ozaydin, Phys. Rev. A 87, 032331 (2013).

[24] C. Yesilyurt, S. Bugu, F. Ozaydin, Quant. Info. Proc. 12, 2965 (2013)

[25] F. Ozaydin, S. Bugu, C. Yesilyurt, A.A. Altintas, M. Tame, S.K. Ozdemir, Phys. Rev. A 89, 042311 (2014).

[26] F. Ozaydin, Phys. Lett. A 378, 3161 (2014). 